\title{
Significance of the hyperfine interactions in the phase diagram of $\mathrm{LiHo}_{\mathrm{x}} \mathrm{Y}_{1-\mathrm{x}} \mathrm{F}_{4}$
}

\author{
M. Schechter and P. C. E. Stamp \\ Department of Physics 83 Astronomy and Pacific Institute for Theoretical Physics, \\ University of British Columbia, Vancouver, British Columbia, Canada V6T $1 Z 1$
}

\begin{abstract}
We consider the quantum magnet $\mathrm{LiHo}_{\mathrm{x}} \mathrm{Y}_{1-\mathrm{x}} \mathrm{F}_{4}$ at $x=0.167$. Experimentally the spin glass to paramagnet transition in this system was studied as a function of the transverse magnetic field and temperature, showing peculiar features: for example (i) the spin glass order is destroyed much faster by thermal fluctuations than by the transverse field; and (ii) the cusp in the nonlinear susceptibility signaling the glass state decreases in size at lower temperature. Here we show that the hyperfine interactions of the Ho atom must dominate in this system, and that along with the transverse inter-Ho dipolar interactions they dictate the structure of the phase diagram. The experimental observations are shown to be natural consequences of this.
\end{abstract}

$\mathrm{LiHo}_{\mathrm{x}} \mathrm{Y}_{1-\mathrm{x}} \mathrm{F}_{4}$ is widely considered to be a model quantum Ising magnet. The strong easy axis crystal field gives the large spin Ho ion an Ising character, behaving as a 2-level system when temperature $T \ll 10 \mathrm{~K}$; quantum fluctuations between the 2 Ising states are induced by a tunable transverse magnetic field $H_{\perp}$. If one then neglects both hyperfine (hf) and transverse dipolar (TD) interactions, the system is described by a transverse field Ising model (TFIM). Experimentally, for $x=1$ the system orders ferromagnetically at low $T$ and $H_{\perp}[1]$; when $x<1$ the longitudinal dipolar interactions become random and frustrated, and for $x=0.167$ it was observed that the system orders in a spin glass (SG) state 2, 3]. Much of the recent theoretical interest in quantum spin glasses [4, [5, [6] has been driven by these experiments [2, 3], and both general theories on quantum spin glasses (see e.g. Ref. 7] ) and specific models of the quantum Ising SG [8, 9] are commonly checked against their results.

However, for $x=0.167$ the hf interaction is larger than the mean dipolar interaction, and in part (i) of the Letter we show that it actually dominates the low-energy physics in this system. The longitudinal hf term forces the two ground doublet states to have a definite, and opposite, nuclear spin 10, 11]. Low transverse magnetic fields $\left(\mu_{\mathrm{B}} H_{\perp} \ll \Omega_{o}\right.$, where $\Omega_{o}$ is the gap to the first excited electronic state) cannot then couple or induce quantum fluctuations between these Ising-like states, but only renormalize their effective spins (and hence the effective dipolar interaction). Transverse hf interactions can induce fluctuations between these states; however, when $\mu_{\mathrm{B}} H_{\perp} \ll \Omega_{o}$ we see that these fluctuations are very weak. Therefore, at low transverse fields, the lowenergy effective Hamiltonian is given by the classical Ising model, with renormalized parameters. We thus explain why $g \mu_{B} H_{c} \gg k T_{c}$ in the experiment [3], i.e., why the transverse field coupling required to destroy the glass is so much larger than the thermal energy required to do the same: While $T_{c}$ at zero field is given by the mean dipolar interaction, the temperature dependence of the transition line on $H_{\perp}$ is dictated by the larger hf interaction that controls the renormalization of the effective spin, and the critical transverse field is controlled by the even larger energy scale of $\Omega_{o}$.
To completely understand how fluctuations are switched on by transverse fields, we show that TD terms must be included. By doing this in a simple way we obtain a satisfactory quantitative agreement with the experimental phase diagram. We then consider the diminishing of the cusp in the nonlinear susceptibility $\chi_{3}$, found in the experiment [3] as temperature is reduced. A natural consequence of our theory is that this may be a result of the renormalization of the effective spin, rather than a first order phase transition.

The Hamiltonian describing the $\mathrm{LiHo}_{\mathrm{x}} \mathrm{Y}_{1-\mathrm{x}} \mathrm{F}_{4}$ system is given by a sum of crystal field 10, 12], Zeeman, hf, and inter-Ho interaction terms:

$$
H=H_{\mathrm{cf}}+H_{\mathrm{Z}}+H_{\mathrm{hf}}+H_{\mathrm{int}} .
$$

The Ho atom has a total angular momentum of $J=8$, and nuclear spin $I=7 / 2$. The Zeeman term $H_{Z}=$ $-\sum_{i} g_{J} \mu_{\mathrm{B}} \vec{H} \cdot \vec{J}_{i}$, and $H_{\mathrm{hf}}=A_{J} \sum_{i} \vec{I}_{i} \cdot \vec{J}_{i}$ is the hf interaction. $H_{\text {int }}=-\sum_{i j} U_{i j}^{\alpha \beta} J_{i}^{\alpha} J_{j}^{\beta}$ is dominated by the dipolar interaction 12]. The interaction of the external magnetic field with the nuclear spins is small, and is neglected here.

The crystal field $H_{c f}$ leaves only three electronic states at low energy; a low-energy doublet, denoted $|\uparrow\rangle$ and $|\downarrow\rangle$ here, and an excited state $\left|\Gamma_{2}^{l}\right\rangle$ roughly $\Omega_{0}=10.5 \mathrm{~K}$ above these. Because of the strong crystal anisotropy the TD and hf terms are usually neglected. Neglecting the longitudinal hf interactions as well, and in the presence of transverse magnetic field $H_{\perp}$, the low-energy effective Hamiltonian for the system would then be the simple TFIM[1]:

$$
H=-\sum_{i, j} V_{i j}^{z z} \tau_{i}^{z} \tau_{j}^{z}-\Delta_{o}\left(H_{\perp}\right) \sum_{i} \tau_{i}^{x},
$$

where $\vec{\tau}_{j}$ is a Pauli vector describing the two-level effective electronic spin at spatial position $\mathbf{r}=\mathbf{r}_{j}$, the $j$-th site, $V_{i j}^{z z} \approx 150 U_{i j}^{z z}$ (with $\left|V_{i j}^{z z}\right| \sim 0.3 \mathrm{~K}$ for nearestneighbour Ho spins), and $\Delta_{o}$ is the transverse fieldinduced coupling between the eigenstates $|\uparrow\rangle$ and $|\downarrow\rangle$ of $\hat{\tau}^{z}$. For small fields $\Delta_{o} \propto H_{\perp}^{2}$ coming from the second-order coupling, mainly via the state $\left|\Gamma_{2}^{l}\right\rangle$ [so that $\left.\Delta_{o} \sim 9\left(\mu_{B} H_{\perp}\right)^{2} / \Omega_{o}\right]$. In this simple model quantum 


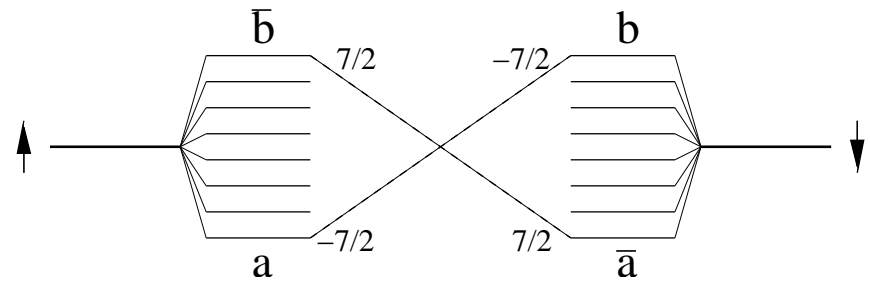

FIG. 1: Splitting of the electronic low-energy doublet ( $\uparrow$ and $\downarrow)$ due to the longitudinal hyperfine interaction. The ground state doublet, $a$ and $\bar{a}$ have a definite and opposite nuclear spin, $\pm 7 / 2$. Transverse magnetic field couples states with the same nuclear spin, as is shown by the dashed lines.

fluctuations are already important at fairly small transverse fields, and we expect a $T=0$ quantum phase transition (QPT) when $\Delta_{o} \sim V_{o}$, where $V_{o} \equiv\left\langle\sum_{j} V_{i j}^{z z}\right\rangle$ is the average dipolar interaction.

For the undiluted system, $x=1$, it was indeed shown 1, 12, 13] that the ferromagnetic paramagnetic (PM) transition line is well described by this model, with a low temperature correction due to hf interactions. However, in the dilute case $V_{o}$ is reduced by a factor $x$, so the $\mathrm{hf}$ interaction is much larger than $V_{o}$. The model (2) is then inadequate, and we must include the hf interactions from the beginning. We now introduce the interactions neglected in the model (2) consecutively, emphasizing the significance of each on the structure of the phase diagram.

(i) Hyperfine Interactions: We first consider the regime $\mu_{\mathrm{B}} H_{\perp} \ll \Omega_{o}$ in which the transverse hf interactions are negligible. The longitudinal hf interaction $H_{\mathrm{hf}}^{z}=A_{J} I^{z} J^{z}$ splits each of the states $|\uparrow\rangle,|\downarrow\rangle$ into an eightfold multiplet of nearly equidistant levels, with separation $\sim 205 \mathrm{mK}[10$ between adjacent levels (Fig (1). The two lowest energy Ising states in zero field are now $a \equiv|\uparrow,-7 / 2\rangle$ and $\bar{a} \equiv$ $|\downarrow, 7 / 2\rangle$, and when $H_{\perp} \neq 0$, these become

$$
|+\rangle=c_{1}|a\rangle+c_{2}|b\rangle \quad ; \quad|-\rangle=c_{1}|\bar{a}\rangle+c_{2}|\bar{b}\rangle,
$$

with coefficients $c_{1}=\alpha \Delta_{o}$, and $c_{2}=\alpha\left[A-\sqrt{A^{2}+\Delta_{o}^{2}}\right]$, where $\alpha=\left[\Delta_{o}^{2}+\left(A-\sqrt{A^{2}+\Delta_{o}^{2}}\right)^{2}\right]^{-1 / 2}$ and $2 A \approx 1.4$ $\mathrm{K}$ is the energy difference between the states $a(\bar{a})$ and $b \equiv|\downarrow,-7 / 2\rangle(\bar{b} \equiv|\uparrow, 7 / 2\rangle)$.

The crucial point here is that the transverse magnetic field $H_{\perp}$ does not couple the relevant Ising doublet states: $a$ and $\bar{a}$ (it actually couples $a$ to $b$ and $\bar{a}$ to $\bar{b}$ ). This immediately invalidates the TFIM (2), since $H_{\perp}$ no longer induces fluctuations. The only effect of the transverse field is to re-normalize the spin: For the state $|+\rangle$ one finds

$$
\left\langle\sigma_{+}^{z}\right\rangle=\eta\left\langle\sigma_{+}^{z}(0)\right\rangle \quad ; \quad \eta \equiv\left(c_{1}^{2}-c_{2}^{2}\right),
$$

and $\left\langle\sigma_{-}^{z}\right\rangle=-\left\langle\sigma_{+}^{z}\right\rangle$. One can then absorb the renormalization into the dipolar interaction, and therefore our system reduces at low transverse fields to the classical Ising model:

$$
H_{\mathrm{eff}}^{\|}=-\sum_{i, j} \tilde{V}_{i j}^{z z} s_{i}^{z} s_{j}^{z}
$$

where $s$ is a spin half matrix in the space of the states $|+\rangle$ and $|-\rangle$ and $\tilde{V}_{i j}^{z z}=\eta^{2} V_{i j}^{z z}$.

As a result, the SG paramagnet transition line is given (in this model, neglecting the later to be shown significant TD interactions) by the relation $T_{c}=\eta^{2} T_{c}(0)$, and the dependence on the transverse field is through $\eta\left(\Delta_{o}\right)$. Importantly, this result is independent of the approximation used to treat the longitudinal dipolar interaction, and relies only on the assertion that $T_{c}$ scales linearly with the interaction. For $\Delta_{o} \ll A$ one has from (44) that $\eta=1-\frac{\Delta_{o}^{2}}{2 A^{2}}$, and defining $\epsilon \equiv\left(T_{c}-T\right) / T_{c}$ one finds that for $\Delta_{o} / A, \epsilon \ll 1$ (i.e., small $H_{\perp}$ and $T \sim T_{c}$ ) the phase transition line $\Delta_{c}(T)$ obeys the relation $\Delta_{c}=A \sqrt{\epsilon}$. For the initial TFIM (2) one would instead get $\Delta_{c} \sim V_{o} \sqrt{\epsilon}$. The difference arises because the hf energy scale $A$ dictates the reduction of the effective spin (and equivalently the effective dipolar interaction). At $T=T_{c} / 2$ one finds that $\Delta_{c} \approx A$, and for $\Delta_{o} / A \gg 1$ we have $\eta=A / \Delta_{o}$, so that $\Delta_{c}=A \sqrt{\left(V_{o} / T\right)}$. Thus, as long as the transverse hyperfine interactions are negligible $\left(\mu_{\mathrm{B}} H_{\perp} \ll \Omega_{o}\right)$ there is no $Q P T$.

We now turn to the discussion of quantum fluctuations in this system, and we thus consider the transverse $\mathrm{hf}$ coupling $H_{\mathrm{hf}}^{\perp}=A_{J}\left(I_{+} J_{-}+I_{-} J_{+}\right) / 2$. This term changes the $z$ component of the nuclear spin by coupling the electronic states $|\uparrow\rangle$ and $|\downarrow\rangle$ with (mainly) the state $\left|\Gamma_{2}^{l}\right\rangle$ at energy $\Omega_{o}$, thus inducing fluctuations between $|+\rangle$ and $|-\rangle$. However, unless $\xi \equiv\left\langle\uparrow\left|\mu_{\mathrm{B}} H_{\perp}\right| \Gamma_{2}^{l}\right\rangle \sim \Omega_{o}$ (corresponding to a field $H_{\perp} \sim 2 \mathrm{~T}$ ), these fluctuations are much smaller than $V_{o}$, and cannot induce a QPT. To see this more quantitatively we diagonalize the single Ho Hamiltonian in a transverse magnetic field, i.e., we diagonalize $H=H_{c f}+H_{Z}+H_{\mathrm{hf}}$, in the 136 eigenfunction space (17 crystal field states $* 8$ nuclear states) using the parameters used in Ref. 12]. We then plot in Fig. 2 the results for the splitting $\tilde{\Delta}\left(H_{\perp}\right)$ between the two lowest levels induced by the combination of $H_{\perp}$ and $H_{\mathrm{hf}}^{\perp}$. For $H_{\perp} \lesssim 3 \mathrm{~T}$ the two lowest levels are well separated from the higher energy levels, and we can replace the classical Ising Hamiltonian (5) by an effective Hamiltonian

$$
H=-\sum_{i, j} \tilde{V}_{i j}^{z z}\left(H_{\perp}, A\right) s_{i}^{z} s_{j}^{z}-\tilde{\Delta}\left(H_{\perp}, A, \Omega_{o}\right) \sum_{i} s_{i}^{x} .
$$

We then expect a zero temperature QPT when $\tilde{\Delta}\left(H_{\perp}\right) \rightarrow$ $\Delta_{c}\left(H_{\perp}\right)$, such that $\Delta_{c}\left(H_{\perp}\right) \approx V_{o}\left(H_{\perp}\right)$. From Fig. 2 we confirm that this happens when $H_{\perp} \sim 2 \mathrm{~T}$. Note there are now three significant energy scales in the problem. $T_{c}$ is dictated by $V_{o}$, while $\Delta_{c}$ is related to both $A$, which dictates the renormalization of the effective dipolar interaction, and to $\Omega_{o}$. Interestingly, for any practical dilution $x$ the lower bound for the critical transverse magnetic field is set by $\Omega_{o}$, since only at $\xi \approx \Omega_{o}$ do quantum fluctuations between the relevant Ising states become appreciable.

We may summarize our analysis so far by saying that the longitudinal hf interactions invalidate the usual quantum Ising model for the $\mathrm{LiHo}_{\mathrm{x}} \mathrm{Y}_{1-\mathrm{x}} \mathrm{F}_{4}$ system, instead creating a classical Ising model whose energetics 
is determined by the longitudinal dipolar interactions, renormalized by longitudinal hf interactions. Adding in the transverse hf interactions brings back an effective transverse field term $\tilde{\Delta}\left(H_{\perp}\right)$ into the effective Hamiltonian, but $\tilde{\Delta}\left(H_{\perp}\right)$ does not become important until much higher transverse fields than in the original model (and it switches on with field in a quite different way from the usual form $\Delta_{o} \propto H_{\perp}^{2}$ ). We emphasize that $\tilde{\Delta}$ should be used instead of $\Delta_{o}$ in analysis of the phase transition and, in particular, for the determination of the critical exponents in the system.

Can our simple model approximation explain the experimental phase diagram? Since according to our analysis up to now, the position of the phase transition line is mainly dictated by single atom properties and the hf interaction, we now derive a phase diagram treating the single Ho exactly, and the inter-Ho interactions by using a mean-field (MF) approximation in which each spin feels an interaction strength proportional to its average $J_{z}$, i.e., using the MF Hamiltonian

$$
\begin{aligned}
H_{\mathrm{MF}}=H_{\mathrm{cf}} & -\sum_{j} g_{J} \mu_{B} H_{\perp} J_{j}^{x} \\
& +A_{J} \sum_{j} \overrightarrow{I_{j}} \cdot \overrightarrow{J_{j}}+\sum_{j} V_{o}\left\langle J_{j}^{z}\right\rangle J_{j}^{z} .
\end{aligned}
$$

In Fig. 3] we plot by dashed line the SG-PM transition obtained within this approximation. $V_{o}$ is fixed by the experimental value of $T_{c}$ at zero transverse field. In order to compare our results to Fig. 1 of Ref. [3] we plot the phase diagram as function of $T$ and $\Delta_{o}$, which is given by the splitting of the electronic levels when the $\mathrm{hf}$ interactions are neglected.

This mean field result does capture two main features of the experiments, viz. (i) the much larger energy associated with quantum disordering of the SG state than with temperature disordering, and (ii) the roughly straight transition line in the $\Delta_{o}, T$ plane in most of the parameter regime. However two noticeable differences are apparent, viz., (i) the experimental $T=0$ quantum critical point occurs at lower transverse field and (ii) the shape of the experimental transition line near the zero field transition point is quite different from the predicted square root behavior. These differences are not a result of our MF approximation. As discussed above, the square root behavior at low transverse fields is a direct consequence of the renormalization of the spin by the transverse magnetic field, and is a single atom property. Furthermore, the experimental graph suggests that the quantum fluctuations induced by the transverse hf interactions are significant already at fields of the order of 1 Tesla, in contrast with Fig 2

(ii) Transverse Dipolar Interactions: The differences just noted suggest that the TD interactions [e.g., $(\alpha \beta)=$ $(z x)$ ], neglected in the Hamiltonian (7), are also significant in the diluted $\mathrm{LiHo}_{\mathrm{x}} \mathrm{Y}_{1-\mathrm{x}} \mathrm{F}_{4}$ system. Unlike for $x=1$, where the transverse terms cancel by symmetry, in the diluted system this is not the case- the TD

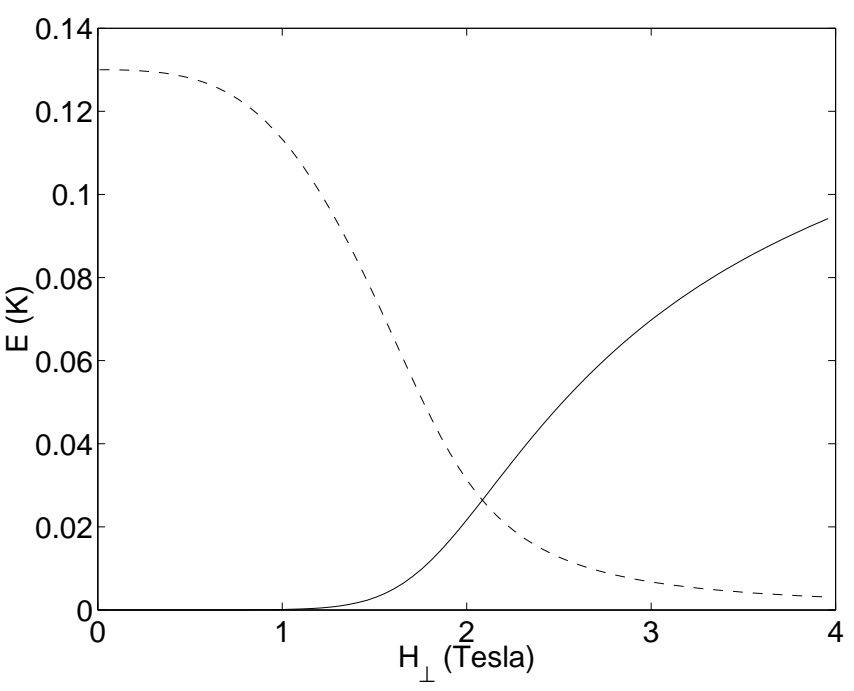

FIG. 2: The splitting $\tilde{\Delta}\left(H_{\perp}, A, \Omega_{o}\right)$ between the ground and first excited state of the single-ion Hamiltonian as a function of $H_{\perp}$ (solid line) calculated by exact diagonalization. This measures the quantum fluctuations between the states $|+\rangle$ and $|-\rangle$. The dashed line is the averaged longitudinal dipolar interaction, taking $V_{o}=0.13$ for $H_{\perp}=0$ to match the experimental $T_{c}$ at zero field.

terms add an effective magnetic field at each Ho location. This field adds both an effective random longitudinal term at each site [14], and a transverse term which can induce fluctuations even in the absence of $H_{\perp}$. We consider the latter effect here, and the former in connection with the nature of the phase transition below. For $\mu_{\mathrm{B}} H_{\perp} \approx V_{o}$ or larger it would seem that the induced transverse fields are random and could enhance or decrease the effective transverse field at each location. However, the external magnetic field breaks time reversal symmetry, and the configurations in which the TD interactions enhance the effective magnetic field at the Ho sites are energetically favorable, as can easily be seen by comparing the two Ho states $|\uparrow, \rightarrow\rangle$ and $|\downarrow, \rightarrow\rangle$. Note, that local strain fields result in random effective fields in the transverse direction as well. However, their magnitude can be estimated to be smaller than that of the TD interactions 10], and more importantly, they are symmetrically distributed with zero mean. Their affect on the effective transverse field is therefore neglected here.

In order to demonstrate the significance of the TD interaction, we now redo the MF calculation with the simplified approximation of taking the effective transverse field as $\tilde{H}_{\perp}=H_{\perp}+H_{\perp}^{d}$. The effective field due to the dipolar interaction $H_{\perp}^{d}$ is the only free parameter in our calculation. The solid line in Fig. 3 is a plot of the SGPM phase transition line including the TD interaction, with $H_{\perp}^{d}=0.65 \mathrm{~T}$. 15] The experimental transition line is reproduced satisfactorily, including its shape near $T_{c}$ and the critical transverse field at low $T$, using a value of $H_{\perp}^{d}$ that is of the order of the typical effective transverse field produced by the TD interactions in the system. Note 


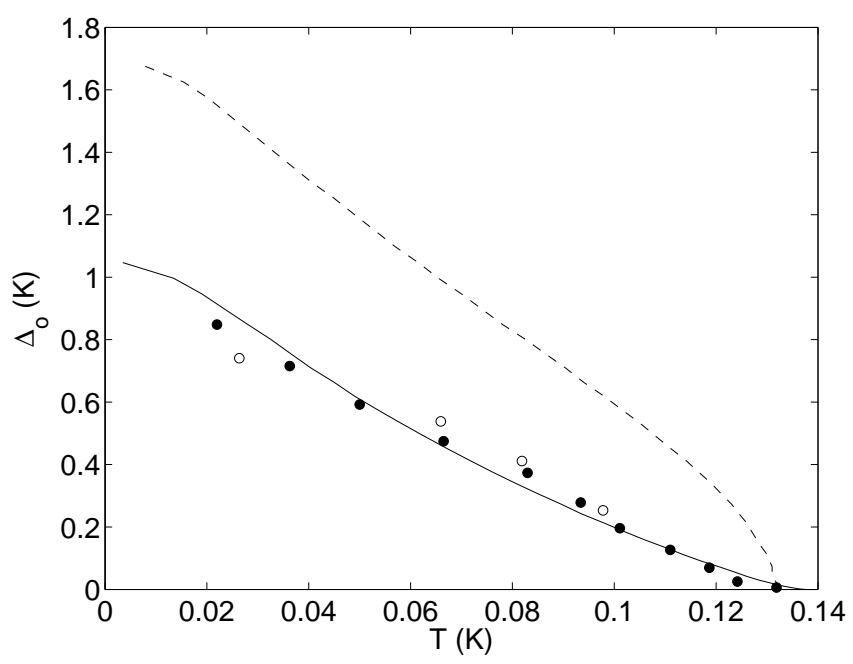

FIG. 3: Spin glass - paramagnet phase transition line in the transverse coupling - temperature $\left(\Delta_{o}, T\right)$ plane, as calculated for the Hamiltonian in Eq. (7) (dashed line) and including the effective field produced by the off-diagonal dipolar interactions (solid line). Circles denote experimental data taken from Fig.1 of Ref. [3].

that our analysis suggests that it is not sufficient to treat the TD interactions in MF, i.e., to replace, e.g., $J_{i}^{z} J_{j}^{x}$ by $J_{i}^{z}\left\langle J_{j}^{x}\right\rangle+\left\langle J_{i}^{z}\right\rangle J_{j}^{x}$. Only the second term potentially adds to the effective transverse field, but at the transition there is no such effect in this approximation since $\left\langle J_{i}^{z}\right\rangle=0$.

Finally, we consider the reduction of the cusp in the nonlinear susceptibility $\chi_{3}(T)$ at low $T$, found experimentally by $\mathrm{Wu}$ et al. (see Fig. 3 of Ref. [3]). This result was interpreted as evidence of a first order phase transition. However, the experimental results should be reconsidered in view of the significance of the hf interactions, leading to Eq. (6), and of the TD interaction. Within the model (6) the transition is expected to be second order. However, by taking into account the electro-nuclear nature of the Ising states and the renormalization of the spin, the diminishing of the cusp is naturally explained: $\chi_{3}(T)$ is conjugate to the Edwards-Anderson parameter, and is proportional [16] to $\sum_{i j}\left\langle S_{i} S_{j}\right\rangle^{2}=S_{0}^{4} \sum_{i j}\left\langle s_{i} s_{j}\right\rangle^{2}$, where $S_{0}$ is the effective electronic spin of the state $|+\rangle$. Experimentally, we assume that the transition point can be approached with a certain accuracy, giving a maximum value to $\sum_{i j}\left\langle s_{i} s_{j}\right\rangle^{2}$; this would result in a maximum value $\operatorname{Max}\left(\chi_{3}(T)\right) \propto S_{0}^{4}$. As $T$ is reduced $H_{c}(T)$ increases, thereby reducing $S_{0}$ and $\operatorname{Max}\left(\chi_{3}(T)\right)$, until the cusp can not be experimentally resolved from the background. In order to establish a different scenario for the phase transition one must treat properly the TD interactions. In Ref. [17] it was shown that a random longitudinal field smears the SG-PM transition of the quantum Ising model, and we expect the TD interaction to do the same.

In this letter we have shown that the usual description of the $\mathrm{LiHo}_{\mathrm{x}} \mathrm{Y}_{1-\mathrm{x}} \mathrm{F}_{4}$ system, in which both hyperfine and TD interactions are neglected, is not adequate to explain the SG-PM phase transition. The large longitudinal hf interactions of the Ho ion force the relevant Ising doublet to be an electro-nuclear state, with definite and opposite values of the spin-7/2 nuclear spins. If it were not for transverse hf interactions, the system would then actually behave like a classical Ising model with renormalized parameters. The quantum nature of the system is revealed only at large transverse fields, proportional to the energy gap to the first excited electronic state, where quantum fluctuations induced by the transverse hf interactions become significant. The TD interaction serves to enhance the effective transverse field, therefore reducing the critical field of the transition. A further study of the role of the TD interactions would be of interest, since our approximate MF treatment does not incorporate multi-spin fluctuations [18]. Recently many interesting phenomena, some connected to the nuclear spins in the system, were observed in the $\mathrm{LiHo}_{\mathrm{x}} \mathrm{Y}_{1-\mathrm{x}} \mathrm{F}_{4}[19,20,21]$. Our approach to the hf interactions applies to all values of dilution at low $T$, and we hope it will help in understanding these phenomena as well.

It is a pleasure to thank Gabriel Aeppli, Michel Gingras, Steve Girvin, Nicolas Laflorencie, Andrea Morello, Subir Sachdev, and Igor Tupitsyn for useful discussions. This work was supported by NSERC in Canada, and by PITP.
[1] D. Bitko, T. F. Rosenbaum, and G. Aeppli, Phys. Rev. Lett. 77, 940 (1996).

[2] W. Wu et al., Phys. Rev. Lett. 67, 2076 (1991).

[3] W. Wu, D. Bitko, T. F. Rosenbaum, and G. Aeppli, Phys. Rev. Lett. 71, 1919 (1993).

[4] J. Miller and D. A. Huse, Phys. Rev. Lett. 70, 3147 (1993); M. Guo, R. N. Bhatt, and D. A. Huse, Phys. Rev. Lett. 72, 4137 (1994); H. Rieger and A. P. Young, Phys. Rev. Lett. 72, 4141 (1994); M.J. Thill and D. A. Huse, Physica A 214, 321 (1995); M. Guo, R. N. Bhatt, and D. A. Huse, Phys. Rev. B 54, 3336 (1996).
[5] Spin Glasses and Random Fields, edited by A. P. Young (World Scientific, Singapore, 1997).

[6] S. Sachdev, Quantum Phase Transitions (Cambridge University Press, Cambridge, 1999).

[7] L. F. Cugliandolo, D. R. Grempel, and C. A. da Silva Santos, Phys. Rev. Lett. 85, 2589 (2000), Phys. Rev. B 64, 014403 (2001).

[8] B. Boechat, R. R. dos Santos, and M. A. Continentino, Phys. Rev. B 49, R6404 (1994).

[9] M. A. Continentino, B. Boechat, and R. R. dos Santos, Phys. Rev. B 50, 13528 (1994). 
[10] R. Giraud et al., Phys. Rev. Lett. 87, 057203 (2001).

[11] B. Barbara et al., J. Magn. Magn. Mater. 272-276, 10241029 (2004).

[12] P. B. Chakraborty et al., Phys. Rev. B 70, 144411 (2004).

[13] V. Banerjee and S. Dattagupta, Phys. Rev. B 64, 024427 (2001).

[14] M. J. P. Gingras (private communication).

[15] The transverse dipolar interactions reduce $T_{c}$ at $H_{\perp}=0$ and we therefore set $V_{o}=0.14$ for $H_{\perp}^{d}=0.65 T$.

[16] K. H. Fischer and J. A. Hertz, Spin glasses (Cambridge
University Press, Cambridge, 1991).

[17] R. Pirc, B. Tadic, and R. Blinc, Phys. Rev. B 36, 8607 (1987).

[18] R. Giraud, A. M. Tkachuk, and B. Barbara, Phys. Rev. Lett. 91, 257204 (2003).

[19] J. Brooke et al., Science 284, 779 (1999)

[20] S. Ghosh et al., Science 296, 2195 (2002)

[21] H. M. Ronnow et al., Science 308, 389 (2005) 